\title{
Centrifuge scaling considerations for fluid-particle systems
}

\author{
T.-S. TAN* and R. F. SCOTT*
}

Two simulations are involved when a centrifuge is used to test models. First, the behaviour of the model in a uniform $n g$ field is assumed to be similar to that of the prototype. Then the centrifuge is assumed to produce an equivalent $n g$ gravitational field. For most static problems, the centrifuge does produce an equivalent $n g$ gravitational field, but for some dynamic problems involving saturated soil these assumptions can break down. When the soil particles and fluid are moving relative to one another, the behaviour in the $n g$ field is not similar to that in the $1 g$ field unless the Reynolds number in both conditions is less than unity. Since this is a special circumstance, the centrifugal behaviour is not similar to that of the prototype in most cases. To illustrate this, the similarity requirements are examined for a single particle moving in a fluid. If different fluids are used in the model and prototype, then the difference in densities must also be accounted for.

Les essais de modèles dans une centrifugeuse impliquent deux simulations. Premièrement, on suppose que le comportement du modèle dans un champ uniforme $n g$ est similaire à celui du prototype. Ensuite, on admet que la centrifugeuse produit un champ gravitationel équivalent à ng. Dans la plupart des cas, la centrifugeuse produit éffectivement un champ gravitationel équivalent à $n g$ mais pour certains problèmes dynamiques concernant des sols saturés ces hypothèses peuvent être erronées. Le comportement à l'intérieur de ce champ n'est analogue à celui de $1 g$ que si le nombre de Reynolds est inférieur à l'unité. Le comportement à l'intérieur de la centrifugeuse n'est pas semblable à celui du prototype. $\mathrm{Si}$ on utilise différents fluides pour le modèle et le prototype, alors il faut tenir compte de la différence de densité.

KEYWORDS: centrifuge modelling; dimensional analysis; liquefaction; sands.

\section{NOTATION}

$a$ radius of the particle

$C_{\mathrm{D}}$ drag coefficient

$g$ acceleration of earth's gravitational field $\left(9.81 \mathrm{~m} / \mathrm{s}^{2}\right)$

$h$ head

$i \quad \partial h / \partial x$, hydraulic gradient

$k \gamma K / \mu$, permeability

$K$ material permeability constant

Discussion on this Paper closes 1 April 1986. For further details see inside back cover.

* California Institute of Technology.

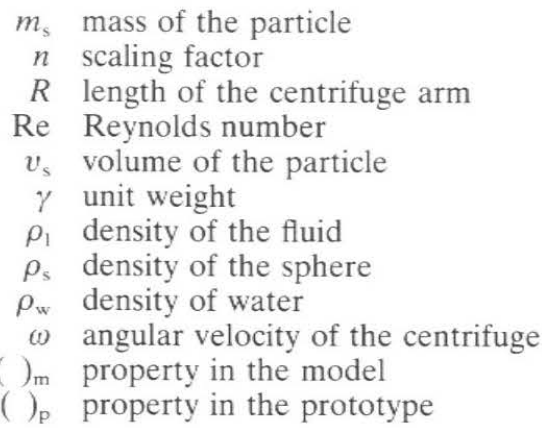

\section{INTRODUCTION}

The difficulties of testing models of soil structures are well known. Often the solution is to test the model in a centrifuge. In recent years, researchers have used the centrifuge to study a variety of soil problems including liquefaction produced by the application of static or dynamic cyclic stresses. Scaling relations have been established for such experiments on the basis of the assumption that the model in the centrifuge is actually subjected to $n$ times the gravitational acceleration of the prototype. There are two parts to this assumption. First, it has to be assumed that the behaviour of the model in an ideal $n g$ gravitational field (the surface of an $n g$ planet) is similar to that of the $1 g$ prototype. Then it must be assumed that the centrifuge is indeed producing an equivalent $n g$ gravitational field.

However, in the study of problems involving relative particle-fluid motion, such as liquefaction, using a centrifuge, the scaling relations pose a problem. Suppose that the linear scaling factor is $n$, i.e. the model is subjected to an acceleration of $n$ times the gravitational. According to current understanding, pore pressure dissipation occurs $n$ times faster than the dynamical process (Table 1 ). This presents a difficulty with some soils if the pore pressures are simultaneously generated dynamically and dissipated by diffusion.

Since diffusion depends on the viscosity of the fluid, one current practice is to use a model fluid of viscosity $n$ times that of the prototype. With 
Table 1. Currently used scaling relations

\begin{tabular}{l|c|c}
\hline \multicolumn{1}{c|}{ Quantity } & $\begin{array}{c}\text { Full scale } \\
\text { (prototype) }\end{array}$ & $\begin{array}{c}\text { Centrifuge } \\
\text { model at } n g\end{array}$ \\
\hline \begin{tabular}{l|c} 
Linear dimension \\
Acceleration
\end{tabular} & 1 & $1 / n$ \\
Velocity (dynamic) & 1 & $n$ \\
Time & & 1 \\
In dynamic terms & 1 & $1 / n$ \\
In diffusion cases & 1 & $1 / n^{2}$ \\
\hline
\end{tabular}

such a fluid, it is intended that the diffusion process will be slowed down $n$ times. This is a reasonable solution provided that it is assumed that the particles during liquefaction are actually experiencing an acceleration that is $n$ times that of the prototype.

Here an attempt is made to check the validity of this assumption. The philosophical aspects of the modelling problem are discussed first. The crux of the situation is to identify precisely the physical behaviour to be modelled. In this aspect, some of the current problems in deriving the scaling relations are also treated. Next, an analytical study of a simplified situation is carried out. The problem to be analysed is that of a single particle moving in a fluid. In the entire analysis, the particle size is assumed to remain the same in the model and in the prototype; this follows the general practice of using the same soil for both the model and the prototype. Should the particle size be different in the model and in the prototype, the governing equations of motion remain the same, but scaling may be complicated owing to Reynolds number changes during particle motion as seen later. The application of these equations to a single particle gives an indication of the motion of an assembly of soil particles at the moment when the soil structure liquefies.

\section{PROBLEMS OF MODELLING LIQUEFACTION IN A CENTRIFUGE}

Current understanding of complete liquefaction suggests that the particles (fine-medium sand grains) lose contact with each other during the process. Thus, the effective stresses are reduced to zero. The particles are initially suspended in the fluid and thereafter sink. It follows that settlement following liquefaction is not a consolidation process but rather sedimentation. This is the mechanism which is modelled here.

In using a centrifuge to study the behaviour of a structure, an attempt is made to simulate a condition whereby the model is subjected to a uniform $n g$ gravitational field. If the particles are in contact and essentially stationary with respect to the bucket, the discrepancy from this ideal situation is due to the divergence and non-uniformity of the imposed acceleration field. These conditions depend on the radius of the centrifuge arm and the size of the specimen; in most geotechnical centrifuge work they are mentioned but generally are not taken into account quantitatively in the analysis. However, when the particles are moving in a fluid in the centrifuge, the motion must be correctly represented and the difference in behaviour from that in a uniform $g$ field may be quite large. A simple example will illustrate this.

Consider a spherical particle of radius $a$ attached at a distance $R$ from the axis of a centrifuge bucket rotating at angular velocity $\omega$ in a vacuum. The particle is clearly subjected to an absolute radial (horizontal) acceleration of $R \omega^{2}(=n g),{ }^{*}$ as are soil particles in the solid state. When the particle is released from its attachment (liquefaction), it will move in a straight line that is tangential to its orbit if it is free to do so (Fig. 1); it thus ceases to experience the $R \omega^{2}(n g)$ acceleration. For a specific case, the particle's motion relative to the bucket (the modelling reference system) has been calculated from its own path and the bucket trajectory, and is shown in Fig. 2 (the scaling of the axes should be noted). In a uniform gravitational field the particle would

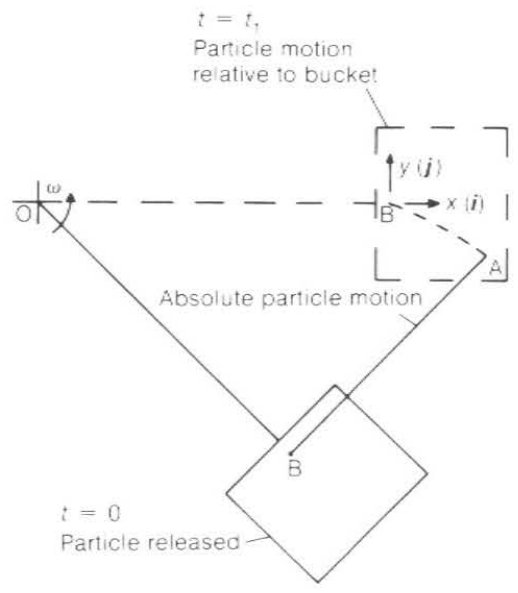

Fig. 1. Reference frame for a particle's motion in a centrifuge

\footnotetext{
* It also obviously experiences the usual vertical gravitational acceleration of $1 \mathrm{~g}$, which is ignored herein in comparison with the other component.
} 


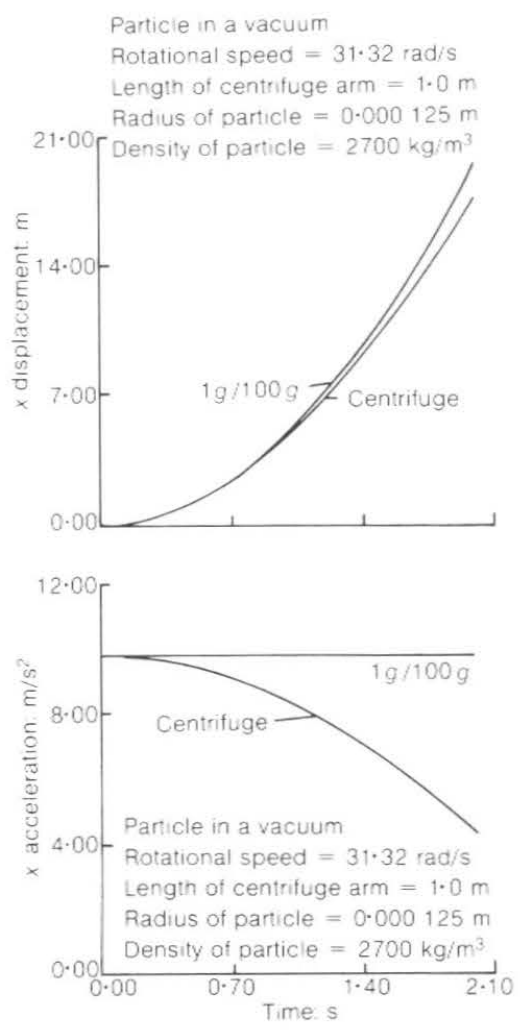

Fig. 2. Particle in a vacuum: behaviours in an $n g$ planet and a centrifuge $\left(\omega^{2} R=n g\right)$ (all quantities shown obey the following relations: time $=($ time in model $) \times n$; acceleration $=($ acceleration in $\operatorname{model}) / n$; displacement $=($ displacement in model $) \times n)$

still be subjected to $n g$ after release and would move in a straight line towards the bottom of the bucket with this acceleration. Fig. 2 shows clearly that this is not the case in the centrifuge. The acceleration, velocity and displacement of the particle with respect to the bucket as a model frame of reference differ from those in the uniform $n g$ field.

Therefore, to derive the true scaling relations for the modelling of liquefaction, the dynamics of a particle moving in a fluid must be examined as a first level of understanding.

To provide for correct scaling relations, it is necessary to ensure that the dynamic quantities, displacement, velocity and acceleration are correctly scaled. Subsequent sections deal with this specifically.

\section{MODELLING POST-LIQUEFACTION IN A CENTRIFUGE}

The problem of modelling the postliquefaction process in a centrifuge is difficult to pose and solve analytically either at the prototype scale or in the centrifuge model. All the particles' interactions, besides the complex problem of each particle's own motion in a fluid, would have to be considered. To gain an insight into the dynamics of particle motion in a fluid, a simplified problem will, instead, be tackled.

Consider a single sphere moving in fluid under two different conditions. They are

(a) a sphere settling in a uniform $n g$ field

(b) a sphere settling in a bucket subjected to centrifugal motion such that the centrifuge acceleration at the initial position of the sphere is $n g\left(\omega^{2} R=n g\right)$.

In both cases, the particle starts from rest with respect to the container surrounding it.

\section{Sphere settling in fluid in uniform ng field (prototype conditions)}

With no initial velocity of the particle relative to the fluid, there is only one space co-ordinate, $x$, and the governing equation of motion for the particle is

$$
\begin{aligned}
v_{\mathrm{s}}\left(\rho_{\mathrm{s}}-\rho_{1}\right) n g-\frac{1}{2} & C_{\mathrm{D}} \rho_{1} \pi a^{2}\left(\frac{\mathrm{d} x}{\mathrm{~d} t}\right)^{2} \\
& =\left(\frac{2}{3} \pi a^{3} \rho_{1}+\frac{4}{3} \pi a^{3} \rho_{\mathrm{s}}\right) \frac{\mathrm{d}^{2} x}{\mathrm{~d} t^{2}}
\end{aligned}
$$

The initial conditions are $x(0)=0, \dot{x}(0)=0$.

In equation (1) the added mass has been taken to be $\frac{2}{3} \pi a^{3} \rho_{1}$ which is the exact value for a sphere accelerating in an ideal fluid. The same term appears in analyses of the hydrodynamic forces in a viscous fluid (Yih, 1977).

For $C_{\mathrm{D}}$, the drag coefficient of a sphere, an approximate fitting of the drag coefficient versus Reynolds number (Re) curve (Schlichting, 1979) was employed to obtain a solution for the motion of the sphere. For different ranges of Reynolds number the fit is given by

$$
\begin{gathered}
\mathrm{Re}<1.0 \\
C_{\mathrm{D}}=24 / \mathrm{Re}
\end{gathered}
$$

where

$$
\begin{gathered}
\operatorname{Re}=\frac{2 \rho_{1} a}{\mu} \frac{\mathrm{d} x}{\mathrm{~d} t} \\
1 \cdot 0 \leqslant \operatorname{Re} \leqslant 2000 \\
C_{\mathrm{D}}=24-14.73 \ln \mathrm{Re}+3 \cdot 669(\ln \mathrm{Re})^{2} \\
-0.4190(\ln \mathrm{Re})^{3}+0.01809(\ln \mathrm{Re})^{4} \\
\operatorname{Re}>2000 \\
C_{\mathrm{D}}=0.4
\end{gathered}
$$


Equation (1) was solved numerically using relations (2), for various cases which will be discussed later.

Sphere in fluid subjected to centrifugal motion

As shown in Fig. 1, A, the position of a particle at time $t_{1}$, at radius $R$, will be observed from a frame of reference (the centrifuge model frame) attached to $\mathrm{B}$, the centrifuge bucket. This frame of reference rotates with the same angular velocity as $\mathrm{B}$ about the axis. The direction $x$ is taken to be perpendicular to the bucket base.

Analysis (Meriam, 1971) gives the acceleration of A, $\boldsymbol{a}_{\mathrm{A}}$, with respect to this frame of reference

$$
\begin{aligned}
& \boldsymbol{a}_{\mathrm{A}}=\left(\ddot{x}-\omega^{2} R-\omega^{2} x-\right.2 \omega \dot{y}) \boldsymbol{i} \\
&+\left(\ddot{y}-\omega^{2} y+2 \omega \dot{x}\right) \boldsymbol{j}
\end{aligned}
$$

The equation of motion is then given by

$$
\begin{array}{r}
m_{\mathrm{s}} \boldsymbol{a}_{\mathrm{A}}+m_{\mathrm{a}}(\ddot{x} \boldsymbol{i}+\ddot{y} \boldsymbol{j})=-\omega^{2} \rho_{1} v_{\mathrm{s}}[(R+x) \boldsymbol{i}+y \boldsymbol{j}] \\
-\frac{1}{2} C_{\mathrm{D}} \rho_{1} \tau a^{2}\left(\dot{x}^{2}+\dot{y}^{2}\right)^{1 / 2}(\dot{x} \boldsymbol{i}+\dot{y} \boldsymbol{j})
\end{array}
$$

The first term on the right-hand side is due to the non-symmetrical pressure distribution on the sphere. It is assumed that the radius of the sphere is very small compared with the length of the centrifuge arm.

Using equation (3) for $\boldsymbol{a}_{\mathrm{A}}$ in equation (4), in the $x$ direction

$$
\begin{aligned}
& \left(\frac{4}{3} \pi a^{3} \rho_{\mathrm{s}}+\frac{2}{3} \pi a^{3} \rho_{1}\right) \ddot{x}+\frac{1}{2} C_{\mathrm{D}} \rho_{1} \pi a^{2}\left(\dot{x}^{2}+\dot{y}^{2}\right)^{1 / 2} \dot{x} \\
& \quad-\omega^{2} v_{\mathrm{s}}\left(\rho_{\mathrm{s}}-\rho_{1}\right) x=v_{\mathrm{s}}\left(\rho_{\mathrm{s}}-\rho_{1}\right) \omega^{2} R+2 \omega \dot{y} m_{\mathrm{s}}
\end{aligned}
$$

and in the $y$ direction

$$
\begin{array}{r}
\left(\frac{4}{3} \pi a^{3} \rho_{\mathrm{s}}+\frac{2}{3} \pi a^{3} \rho_{1}\right) \ddot{y}+\frac{1}{2} C_{\mathrm{D}} \rho_{1} \pi a^{2}\left(\dot{x}^{2}+\dot{y}^{2}\right)^{1 / 2} \dot{y} \\
-\omega^{2} v_{\mathrm{s}}\left(\rho_{\mathrm{s}}-\rho_{1}\right) y=-2 \omega \dot{x} m_{\mathrm{s}}
\end{array}
$$

For comparison with the $n g$ gravitational field solutions, equations (5) and (6) were solved numerically for the drag coefficient functions given in equation (2).

As a rough check on the validity of the solutions, one displacement solution (an $x$ versus $y$ plot) is compared with the actual flight path photographed at the Tokyo Institute of Technology (Kimura, Nakase, Kusakabe, Saitoh \& Ohta, 1982) for air as the fluid. The photographed flight path is that of a stream of particles. If there were no particle interaction, and the air were still, then this flight path would be the same as the trajectory of a single particle. Fig. 3 compares the calculated flight path with the actual flight path. It is seen that the expected interactions do not play a substantial

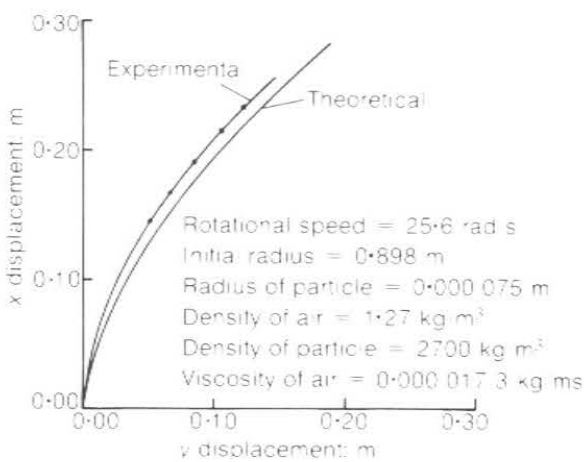

Fig. 3. Particle in air: comparison of experimental flight path and calculated flight path (refer to Fig. 1 for the definitions of $x$ and $y$ )

part in modifying the single particle's trajectory. The curved flight path in this figure illustrates the influence of the Coriolis force on the particle. The Coriolis force (Pokrovsky \& Fyodorov, 1975) is of major importance in centrifuge studies whenever the velocity of the particle is significant. The solution to equations (5) and (6), for general initial conditions, can thus be used in a variety of problems, including, for example, the placement of soil structures in flight and the study of patterns of soil deposits in cratering experiments in the centrifuge.

\section{RELATION OF PARTICLE DYNAMICS TO SCALING RELATIONS}

There are two simulations involved in using a scaled model in a centrifuge to study a prototype structure. First, it is assumed that the model in a uniform $n g$ field behaves in a manner that is similar to the prototype. Then it is assumed that the model in a centrifuge has the same behaviour as that in an $n g$ field. A breakdown in either of these two simulations will render experiments in a centrifuge less meaningful. The relative motion of a particle will again be considered, for comparing the different conditions. In each subsequent solution the particle is considered to be attached to the bucket and is released.

\section{Behaviour of sphere in uniform ng field}

For the model, equation (1) gives

$$
A n g+\left(C_{\mathrm{D}}\right)_{\mathrm{m}} B\left(\frac{\mathrm{d} x_{\mathrm{m}}}{\mathrm{d} t_{\mathrm{m}}}\right)^{2}=C \frac{\mathrm{d}^{2} x_{\mathrm{m}}}{\mathrm{d} t_{\mathrm{m}}{ }^{2}}
$$

where $\quad A=v_{\mathrm{s}}\left(\rho_{\mathrm{s}}-\rho_{1}\right), \quad B=-\frac{1}{2} \rho_{1} \pi a^{2}$, $\mathrm{C}=\frac{2}{3} \pi a^{3}\left(\rho_{1}+2 \rho_{\mathrm{s}}\right)$, and $A, B$ and $C$ have the same values in both the model and the prototype provided that the particle radius and 
particle and fluid densities in the model and the prototype are the same. The drag coefficient will generally be different in the model and the prototype.

Assuming that this is the case at present, then for the prototype $n=1$ and equation (1) becomes

$$
A g+\left(C_{\mathrm{D}}\right)_{\mathrm{p}} B\left(\frac{\mathrm{d} x_{\mathrm{p}}}{\mathrm{d} t_{\mathrm{p}}}\right)^{2}=C \frac{\mathrm{d}^{2} x_{\mathrm{p}}}{\mathrm{d} t_{\mathrm{p}}{ }^{2}}
$$

For the construction of the model, the linear dimension is scaled as $x_{\mathrm{m}} / x_{\mathrm{p}}=1 / n$. Putting this in equation (7) and dividing the result by $n$ gives for the model

$$
A g+\left(C_{\mathrm{D}}\right)_{\mathrm{m}} B \frac{1}{n^{3}}\left(\frac{\mathrm{d} x_{\mathrm{p}}}{\mathrm{d} t_{\mathrm{m}}}\right)^{2}=\frac{C}{n^{2}} \frac{\mathrm{d}^{2} x_{\mathrm{p}}}{\mathrm{d} t_{\mathrm{m}}{ }^{2}}
$$

If $C_{\mathrm{D}}$ or $B=0$, in both the model and the prototype, as is the case for example when the particle moves in a vacuum, then it is observed that equation (9) is similar to equation (8) if $t_{\mathrm{m}} / t_{\mathrm{p}}$ is set equal to $1 / n$. This is the scaling relation that we obtain in the so-called 'dynamic' case.

However, if $C_{\mathrm{D}} \neq 0$ and $B \neq 0$, then for equations (8) and (9) to be equivalent both the following conditions must be met

$$
\begin{aligned}
t_{\mathrm{m}} & =\frac{1}{n} t_{\mathrm{p}} \\
\left(C_{\mathrm{D}}\right)_{\mathrm{m}} & =n\left(C_{\mathrm{D}}\right)_{\mathrm{p}}
\end{aligned}
$$

If the same fluid is used in both the prototype and the model, equation (11) is not satisfied and the two behaviours will not be similar. The deviation in terms of acceleration is shown in Fig. 4, where the same fluid is used in all five cases (for $n$ values of $1,5,10,50,100$ ). To understand this deviation, it is observed from equation (2) that $C_{\mathrm{D}}$, the drag coefficient, is a function of Reynolds number. Since the particle size remains unchanged, the same fluid is used, and the velocity is the same in the model and the prototype (from equation (10) and $x_{\mathrm{m}}=$ $\left.x_{\mathrm{p}} / n\right)$, the Reynolds number is thus not scaled in such a way that equation (11) is satisfied. For correct scaling, it is crucial that equation (11) be obeyed whenever the problem involves a two-phase medium of which one phase is a fluid and the other is solid particles. Liquefaction is only one example of such a situation. More specifically, for very low Reynold's numbers $(\operatorname{Re}<1 \cdot 0)$

$$
C_{\mathrm{D}}=\frac{24}{\operatorname{Re}}=\frac{24 \mu}{2 \rho_{1} a \mathrm{~d} x / \mathrm{d} t}
$$

Noting that, if equation (10) holds, $\mathrm{d} x_{\mathrm{p}} / \mathrm{d} t_{\mathrm{p}}$

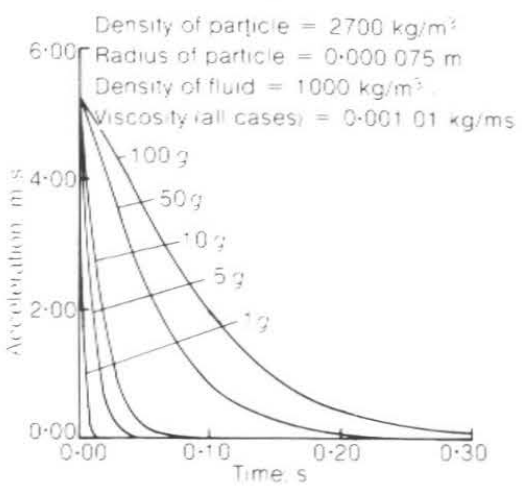

Fig. 4. Behaviour in a uniform $n g$ field ( $n$ values of $1,5,10,50$ and 100): $\mu_{\mathrm{m}}=\mu_{\mathrm{p}}$ (all quantities shown obey the following relations: time $=($ time in model $)$ $\times n ;$ acceleration $=($ acceleration in $\operatorname{model}) / n)$

$=\mathrm{d} x_{\mathrm{m}} / \mathrm{d} t_{\mathrm{m}}$, and inserting equation (12) in equations (7) and (8) respectively the following equations result

(equation (7))

$$
A g+\mu_{\mathrm{p}} \frac{24 B}{2 \rho_{\mathrm{l}} a} \frac{\mathrm{d} x_{\mathrm{p}}}{\mathrm{d} t_{\mathrm{p}}}=C \frac{\mathrm{d}^{2} x_{\mathrm{p}}}{\mathrm{d} t_{\mathrm{p}}{ }^{2}}
$$

(equation (8))

$$
A g+\frac{\mu_{\mathrm{m}}}{n} \frac{24 B}{2 \rho_{1} a} \frac{\mathrm{d} x_{\mathrm{p}}}{\mathrm{d} t_{\mathrm{p}}}=C \frac{\mathrm{d}^{2} x_{\mathrm{p}}}{\mathrm{d} t_{\mathrm{p}}{ }^{2}}
$$

Clearly equations (13) and (14) will be identical if $\mu_{\mathrm{m}}=n \mu_{\mathrm{p}}$. In this case, the drag force is linear with respect to the velocity (second term on the left-hand sides of equations (13) and (14)) and allows similarity to be achieved. Also, the Reynolds number of the particle in the model is smaller than that in the prototype by a factor $n$. Thus, if the Reynolds number in the prototype is less than unity over the entire range of velocities, similarity is retained in the behaviour of the model and the prototype if the model fluid has a viscosity that is $n$ times that of the prototype. This situation would occur for particles of size less than $0.1 \mathrm{~mm}$, when the prototype fluid is water.

It is also worthwhile to point out that the notion that there are two time-scales if the same fluid is used in both the model and the prototype, one for 'dynamic time' and one for 'diffusion time', is incorrect.

It is obvious where this idea comes from. If in transforming equation (9) to equation (14) the following substitutions are made

(a) $\mu_{\mathrm{m}}=\mu_{\mathrm{p}}$

(b) $t_{\mathrm{m}} / t_{\mathrm{p}}=1 / n^{2}$ for the drag term

(c) $t_{\mathrm{m}} / t_{\mathrm{p}}=1 / n$ for the dynamic term 
then equation (14) is indeed similar to equation (13). To be consistent, only one time-scale can be applied to the equation, but in this case the two equations, model and prototype, cannot be similar.

In the more usual case where the size of the particle is greater than $0.1 \mathrm{~mm}$, even if the model fluid viscosity is $n$ times that of the prototype, similarity is achieved only briefly when the Reynolds number of the particle is less than unity in both the model and the prototype. However, when the Reynolds number exceeds unity in the prototype, the drag force becomes non-linear with respect to velocity whereas the drag force in the model is still linear with respect to velocity. Thus similarity cannot be achieved.

For example, consider the case where the particle size is $0.25 \mathrm{~mm}$ (radius $=0.125 \mathrm{~mm}$ ) and $n=100$ (Fig. 5). In this case, the Reynolds number of the particle in the model is less than unity over the range of velocities, reaching a terminal velocity of $0.057 \mathrm{~m} / \mathrm{s}$ and a Reynolds number of $0 \cdot 142$, but in the $1 g$ prototype the Reynolds number of the particle is less than unity for a period shorter than $0.001 \mathrm{~s}$ and finally reaches a terminal value of 7.43 at a velocity of $0.03 \mathrm{~m} / \mathrm{s}$. If there were similarity in behaviour in the model and the prototype, then the velocities shown in Fig. 5 should be identical.

Figures 6-9 portray the effects of changing particle size, with resulting variation in Reynolds number and drag coefficient. These effects are illustrated in terms of the different accelerations as a function of $g$ level and time. In the calculations, $\mu_{\mathrm{m}}$ is set equal to $n \mu_{\mathrm{p}}(n$

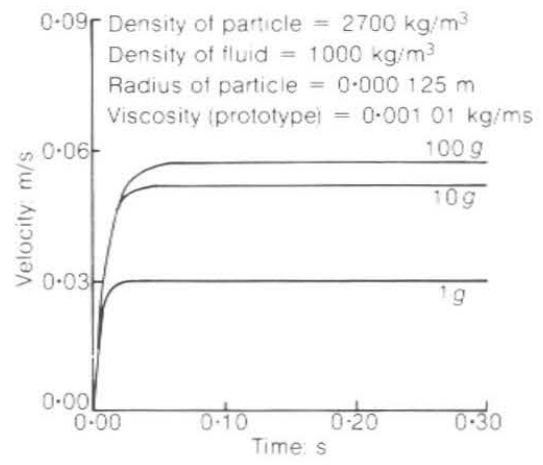

Fig. 5. Behaviour of a particle of diameter $\mathbf{0 . 2 5} \mathrm{mm}$ in a uniform $n g$ field ( $n$ values of 1,10 and 100): $\mu_{\mathrm{m}}=n \mu_{\mathrm{p}} \quad$ (quantities shown obey the following relations: time $=($ time in $\operatorname{model}) \times n ;$ velocity $=$ velocity in model)

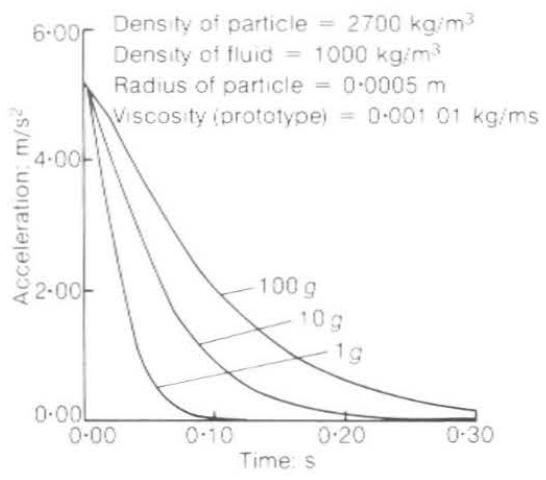

Fig. 6. Behaviour of a particle of diameter $1 \cdot 0 \mathrm{~mm}$ in a uniform $n g$ field ( $n$ values of 1, 10 and 100): $\mu_{\mathrm{m}}=n \mu_{\mathrm{p}}$ (quantities shown obey the following relations: time $=($ time in model $) \times n ;$ acceleration $=($ acceleration in model) $/ n$ )

values of 1,10 and 100) and the fluid densities are assumed to remain the same. The particle diameter in the figures ranges from $1 \mathrm{~mm}$ to $0 \cdot 15 \mathrm{~mm}$. It should be noted that the curves come closer together as the particle size decreases. Complete similarity would require all curves to coincide.

It must be emphasized that these conclusions are derived from relations describing the motion of a single particle in a fluid only. The more general case of interest involves relative movement between the fluid and a mass of particles, in contact with each other or not. This is the condition where Darcy's law is expected to apply. Before liquefaction the particles are in contact, and fluid may be flowing through the granular mass; after liquefaction, the grains are also moving with respect to a fixed reference. Before liquefaction, with the same fluid in the prototype and the centrifuge model, the velocity

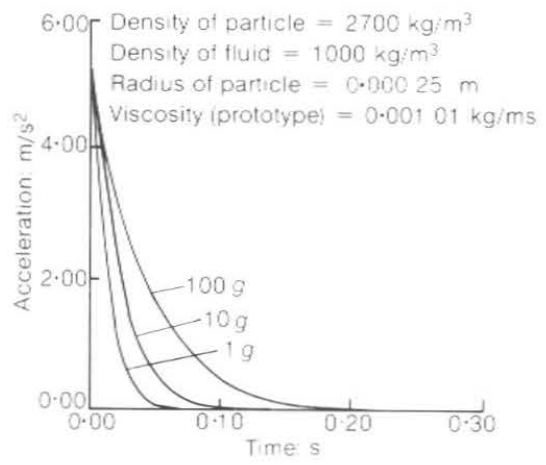

Fig. 7. Behaviour of a particle of diameter $0.5 \mathrm{~mm}$ in a uniform $n g$ field ( $n$ values of 1,10 and 100): $\mu_{\mathrm{m}}=n \mu_{\mathrm{p}}$ (quantities shown are scaled as in Fig. 6) 


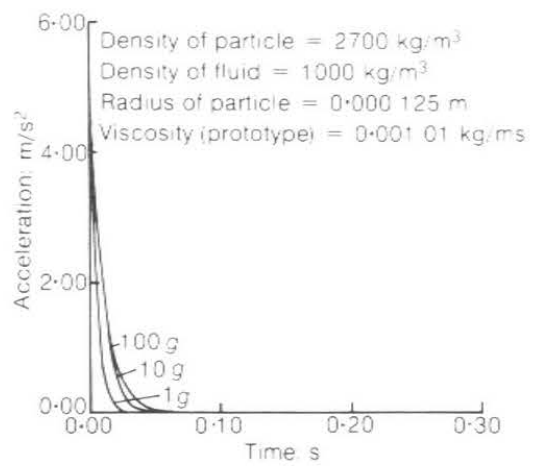

Fig. 8. Behaviour of a particle of diameter $0 \cdot 25 \mathrm{~mm}$ in a uniform $n g$ field ( $n$ values of 1,10 and 100): $\mu_{\mathrm{m}}=n \mu_{\mathrm{p}}$ (quantities shown are scaled as in Fig. 6)

of the fluid with respect to the grains is $n$ times greater in the centrifuge, in which, therefore, the behaviour may no longer correspond to Darcy's law. After liquefaction, the acceleration acting on the soil grains is no longer defined, and thus, whatever fluids are being used, and even if Darcy's law is applicable to both the prototype and the model, similarity no longer holds.

This analysis assumes that the densities of fluids in the model and the prototype are the same, but for the model fluid to have a viscosity that is $n$ times that of the prototype, a glycerine-water mixture may be used, in which case the model fluid density is different from the fluid density in the prototype. Other model fluids, such as silicone oil (Dean \& Schofield, 1983) may be more acceptable in terms of density equivalence. A difference in densities can be significant and has to be accounted for.

Assuming that the fluid in the prototype is

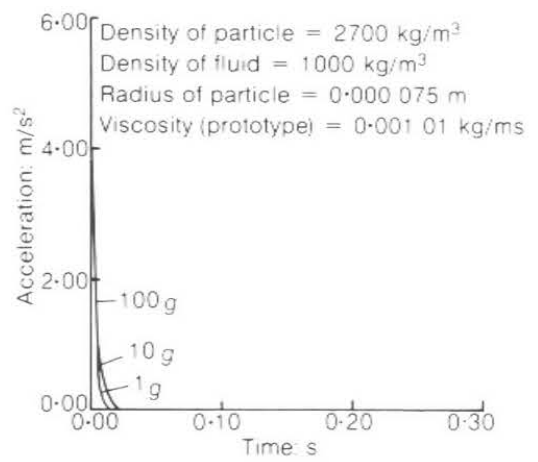

Fig. 9. Behaviour of a particle of diameter $0.15 \mathrm{~mm}$ in a uniform $n g$ field ( $n$ values of 1,10 and 100): $\mu_{\mathrm{m}}=n \mu_{\mathrm{p}}$ (quantities shown are scaled as in Fig. 6) water, equation (1) with $n=1$ and $\rho_{1}=\rho_{\mathrm{w}}$ gives

$$
\begin{aligned}
v_{\mathrm{s}}\left(\rho_{\mathrm{s}}-\rho_{\mathrm{w}}\right) g-\frac{1}{2}\left(C_{\mathrm{D}}\right)_{\rho} \rho_{\mathrm{w}} \pi a^{2}\left(\frac{\mathrm{d} x_{\mathrm{p}}}{\mathrm{d} t_{\mathrm{p}}}\right)^{2} \\
=\frac{v_{\mathrm{s}}}{2}\left(\rho_{\mathrm{w}}+2 \rho_{\mathrm{s}}\right) \frac{\mathrm{d}^{2} x_{\mathrm{p}}}{\mathrm{d} t_{\mathrm{p}}{ }^{2}}
\end{aligned}
$$

For the model

$$
\begin{aligned}
v_{\mathrm{s}}\left(\rho_{\mathrm{s}}-\rho_{\mathrm{l}}\right) n g-\frac{1}{2}\left(C_{\mathrm{D}}\right)_{\mathrm{m}} \rho_{1} \pi a^{2}\left(\frac{\mathrm{d} x_{\mathrm{m}}}{\mathrm{d} t_{\mathrm{m}}}\right)^{2} \\
=\frac{v_{\mathrm{s}}}{2}\left(\rho_{1}+2 \rho_{\mathrm{s}}\right) \frac{\mathrm{d}^{2} x_{\mathrm{m}}}{\mathrm{d} t_{\mathrm{m}}{ }^{2}}
\end{aligned}
$$

If $x_{\mathrm{m}} / x_{\mathrm{p}}=1 / n$, and it is assumed that the Reynolds number is less than unity in both the model and the prototype, then for equations (15) and (16) to be similar, the following requirements must be met

$$
\begin{aligned}
\frac{t_{\mathrm{m}}}{t_{\mathrm{p}}} & =\left[\frac{\left(\rho_{\mathrm{l}}+2 \rho_{\mathrm{s}}\right)\left(\rho_{\mathrm{s}}-\rho_{\mathrm{w}}\right)}{\left(\rho_{\mathrm{w}}+2 \rho_{\mathrm{s}}\right)\left(\rho_{\mathrm{s}}-\rho_{1}\right)}\right]^{1 / 2} \frac{1}{n} \\
\mu_{\mathrm{m}} & =\left[\frac{\left(\rho_{1}+2 \rho_{\mathrm{s}}\right)\left(\rho_{\mathrm{s}}-\rho_{1}\right)}{\left(\rho_{\mathrm{w}}+2 \rho_{\mathrm{s}}\right)\left(\rho_{\mathrm{s}}-\rho_{\mathrm{w}}\right)}\right]^{1 / 2} n \mu_{\mathrm{p}} \\
\frac{\mathrm{d} x_{\mathrm{m}}}{\mathrm{d} t_{\mathrm{m}}} & =\left[\frac{\left(\rho_{\mathrm{w}}+2 \rho_{\mathrm{s}}\right)\left(\rho_{\mathrm{s}}-\rho_{1}\right)}{\left(\rho_{1}+2 \rho_{\mathrm{s}}\right)\left(\rho_{\mathrm{s}}-\rho_{\mathrm{w}}\right)}\right]^{1 / 2} \frac{\mathrm{d} x_{\mathrm{p}}}{\mathrm{d} t_{\mathrm{p}}} \\
\frac{\mathrm{d}^{2} x_{\mathrm{m}}}{\mathrm{d} t_{\mathrm{m}}{ }^{2}} & =\left[\frac{\left(\rho_{\mathrm{w}}+2 \rho_{\mathrm{s}}\right)\left(\rho_{\mathrm{s}}-\rho_{1}\right)}{\left(\rho_{1}+2 \rho_{\mathrm{s}}\right)\left(\rho_{\mathrm{s}}-\rho_{\mathrm{w}}\right)}\right] n \frac{\mathrm{d}^{2} x_{\mathrm{p}}}{\mathrm{d} t_{\mathrm{p}}{ }^{2}}
\end{aligned}
$$

To indicate the consequences of these relations, the case is considered where $\rho_{\mathrm{w}}=$ $1000 \mathrm{~kg} / \mathrm{m}^{3}, \quad \rho_{\mathrm{s}}=2700 \mathrm{~kg} / \mathrm{m}^{3}, \quad \rho_{1}=1220 \mathrm{~kg} / \mathrm{m}^{3}$ and $a=0.05 \mathrm{~mm}$. Equation (17) gives

$$
\frac{t_{\mathrm{m}}}{t_{\mathrm{p}}}=\frac{1}{0 \cdot 917} \frac{1}{n}
$$

and equation (20) gives

$$
\frac{\mathrm{d}^{2} x_{\mathrm{m}}}{\mathrm{d} t_{\mathrm{m}}{ }^{2}}=0.841 n \frac{\mathrm{d}^{2} x_{\mathrm{p}}}{\mathrm{d} t_{\mathrm{p}}{ }^{2}}
$$

In this example, where the particle is small enough to maintain $\operatorname{Re}<1$, it is observed that the acceleration in the model is about $84 \%$ of the currently assumed value of $n$ times the prototype acceleration. In the scaling of time, there is a difference of $8 \%$ from the current assumption. Fig. 10 illustrates the discrepancy if the difference is not accounted for. For larger diameter grains, the deviation is worse. Equations (17), (19) and (20) give the true scaling relations, taking into account the difference in densities. These should be used for correct scaling. Fig. 11 illustrates the improved results when equations (17)-(20) are used. 


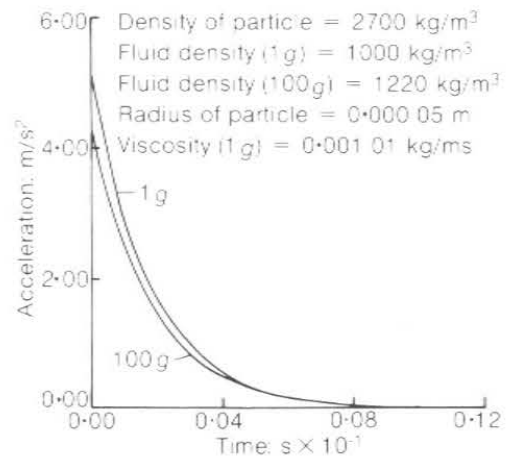

Fig. 10. Behaviour of a particle of diameter $0 \cdot 1 \mathrm{~mm}$ in a uniform $n g$ field ( $n$ values of 1 and 100) (the difference in densities of the fluids is not taken into account: scalings for time, viscosity and acceleration are given by equations (17), (18) and (20) respectively)

\section{Behaviour of sphere in centrifuge}

It is observed from equation (5) that, if $\dot{y} \approx 0$ and $x$ is small compared with $R$, then since $\omega^{2} R=n g$ equation (5) is exactly the same as equation (1). However, when this is not the case, equation (5) can be very different from equation (1) and the centrifuge will not correctly simulate the $n g$ field. Fig. 2 where the 'fluid' is a vacuum clearly illustrates the difference when $\dot{y} \neq 0$ and $x \ll R$.

Calculations show that, in a liquid, grains with the usual density of soil solids move in almost a straight line to the bottom of the container in a centrifuge test, i.e. the $y$ component of motion is not very important.

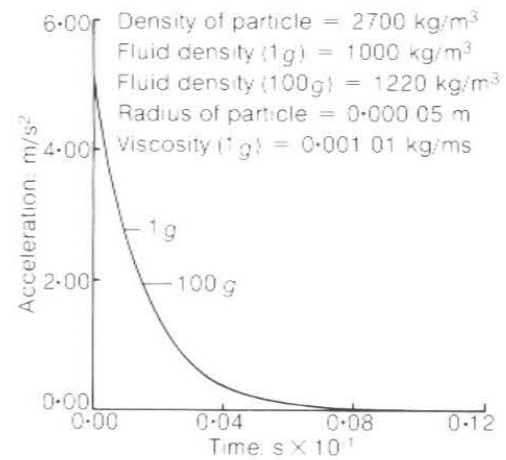

Fig. 11. Behaviour of a particle of diameter $0 \cdot 1 \mathrm{~mm}$ in a uniform $n g$ field ( $n$ values of 1 and 100) (the difference in densities of the fluids is taken into account: scalings for time, viscosity and acceleration are given by equations (17), (18) and (20) respectively)

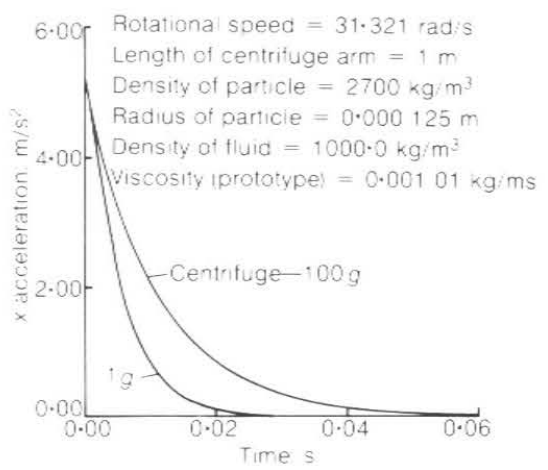

Fig. 12. Behaviour of a particle of diameter $\mathbf{0} \cdot 25 \mathrm{~mm}$ in a uniform $1 \mathrm{~g}$ field, $100 \mathrm{~g}$ field and a centrifuge $\left(\omega^{2} R=100 \mathrm{~g}\right): \mu_{\mathrm{m}}=n \mu_{\mathrm{p}}$ (the density of the fluid is assumed to remain the same; the quantities shown are scaled as in Fig. 6)

However, if, in the centrifuge, the particles are very dense, or if sand grains are moving in air or in a vacuum, the $y$ component is significant, as shown in Fig. 3, and the particle's path is curved.

For a sand particle with a size in the range considered in this Paper in a fluid with viscosity $n$ times that of the prototype, calculations show that the centrifuge is an excellent simulation of the $n g$ field. Fig. 12 shows that the solutions to equation (1) for $n=100$ and to equation (5) for $\omega^{2} R=100 g$ are almost identical. However, this does not mean that the centrifuge is modelling the prototype's behaviour correctly since it has been shown that the $n g$ field is not a good simulation of the $1 g$ field.

Currently, in the special case of relative fluid-particle movement, when fluid is flowing through a granular medium, there are different points of view among various investigators in the derivation of the scaling relations even when the problem raised above does not exist. With respect to centrifuge tests Schofield (1980) says

'The model seepage flow is driven by the full prototype head, but it has only the small scale path length, which is what makes the model hydraulic gradient $n i$ and hence causes the model seepage velocity to be $n$ times the prototype velocity.... The quantity $Q$ seeping into a metre length of tunnel in time $t$ is $Q=\Delta h k t N_{\mathrm{F}} / N_{\mathrm{D}}$ which is the same for model and prototype if model and prototype both have the same permeability.'

Goodings (1984) makes similar statements. 
Darcy's law (Schofield \& Wroth, 1968) gives

$$
v=-k \frac{\partial h}{\partial x}
$$

Now, the permeability $k$ is given by the relation (Lambe \& Whitman, 1979)

$$
k=\frac{\gamma K}{\mu}
$$

Clearly, in this generally accepted definition, $k$ depends on the product $\rho_{1} g$ of the fluid (Terzaghi, 1943; Taylor, 1948), so that

$$
\frac{k_{\mathrm{m}}}{k_{\mathrm{p}}}=\frac{\gamma_{\mathrm{m}}}{\gamma_{\mathrm{p}}}=n
$$

although the original writers were concerned with variations in the density $\rho_{1}$ of the fluid rather than with performing permeability tests using the same fluid in different gravitational fields. With this reasoning, the centrifuge model permeability is $n$ times greater than that of the same soil in the prototype $1 g$ environment. A hydraulic gradient defined in the usual way as a head difference divided by a distance has the same value in the centrifuge model and the prototype since the head (distance) and the length scale in the same way. Therefore, the model fluid velocity $v_{\mathrm{m}}$ is given by the relation

$$
v_{\mathrm{m}}=k_{\mathrm{m}} \frac{\partial h_{\mathrm{m}}}{\partial x_{\mathrm{m}}}=n k_{\mathrm{p}} \frac{\partial h_{\mathrm{p}}}{\partial x_{\mathrm{p}}}=n v_{\mathrm{p}}
$$

It is the increase in permeability as defined by equation (23) in the model that causes its seepage velocity to be $n$ times that of the prototype. Roscoe (1968) addressed the same point, but defined his gradient in terms of pressure. The permeability that he used was thus independent of $\gamma$, the unit weight of the water. Consequently, his definition differs from the current expression for $k$ given by equation (22). In Roscoe's case, the permeability so defined, which has not been used elsewhere in soil mechanics to the Authors' knowledge, remained the same in the model and the prototype and the increase in seepage velocity was accounted for by the increased pressure (not head) gradient.

\section{SUMMARIZING REMARKS}

The analysis presented is simplistic. In the real case, the fact that there are many particles in the fluid has to be considered. The development of pore pressure and its subsequent dissipation is a very complicated process. However, if, during liquefaction, the particles settle rather than behave according to consolidation theory, then this analysis gives a guide to deriving scaling relations for models in a centrifuge.

The conclusions are as follows.

(a) There are two simulations involved in the modelling process. The first is the simulation using a model in a uniform $n g$ field and the second is the use of a centrifuge to produce an $n g$ field. It must be ensured that both simulations produce similar behaviours, otherwise the scaling relations will be wrong. The analyses presented here have demonstrated that, for soil particles larger than $0.1 \mathrm{~mm}$ in water, the centrifuge model does not simulate the prototype behaviour well. However, perhaps contrary to expectations, the breakdown is due to the fact that the behaviour of a particle moving in a fluid in a uniform $n g$ field is not similar to that in a $1 \mathrm{~g}$ field. The difficulty in achieving similarity in the two situations arises because of the complicated nature of fluid behaviour and the non-linearity of the drag coefficient. In most practical cases, the centrifuge is an excellent simulation of the $n g$ field but not of the prototype condition.

(b) It must be realized that in using a model fluid that is $n$ times more viscous than water the density of the fluid may also change and the change needs to be accounted for by using equations (16)-(20) for scaling of dynamic quantities.

(c) The prevailing concept that, if the same fluid is used in both the model and the prototype, there are two time-scales (one for 'dynamic time' and one for 'diffusion time') is incorrect. For there to be similarity between behaviours in the model and the prototype, only one time-scale can be used, which requires that other parameters must be scaled so that the equations are indeed made similar.

(d) The paper by Kimura et al. (1982) demonstrates the difficulty of constructing a dry soil structure while in flight. The difficulty arises mainly because of the effect of the Coriolis force. The solution generated and illustrated in Fig. 3 can be used to design a system for building a desired shape of soil structure in flight.

(e) In general if a structure (grain, section of failing slope) has a significant velocity relative to the bucket the dynamics must include Coriolis effects, and the scaling relation must take this into account (Pokrovsky \& Fyodorov, 1975). 


\section{ACKNOWLEDGEMENTS}

The Authors thank Professor Kimura and Dr Kusakabe of Tokyo Institute of Technology for providing them with a photograph of the flight path of sand particles. A portion of this work was funded by National Science Foundation grant CEE 8219068.

\section{REFERENCES}

Dean, E. T. R. \& Schofield, A. N. (1983). Two centrifuge model tests: earthquakes on submerged embankments. Proc. Atti del XV Convegno Nazionale di Geotecnica, Spoleto, pp. 115-129.

Goodings, D. J. (1984). Relationships for modelling water effects in geotechnical centrifuge models. Proc. Symp. Application of Centrifuge Modelling to Geotechnical Design, Manchester, 16-18 April, pp. 1-24. University of Manchester.

Kimura, T., Nakase A., Kusakabe, O., Saitoh, L. \& Ohta, A. (1982). Geotechnical centrifuge model tests at the Tokyo Institute of Technology.
Technical Report No. 30, Tokyo Institute of Technology.

Lambe, T. W. \& Whitman, R. V. (1979). Soil mechanics SI version, p. 287. New York: Wiley.

Meriam, J. L. (1971). Dynamics, 2nd edn, pp. 64-67. New York: Wiley.

Pokrovsky, G. I. \& Fyodorov, I. S. (1975). Centrifugal model testing in the construction industry, vol. 1. Garston: Building Research Establishment.

Roscoe, K. H. (1968). Soils and model tests. J. Strain Anal. 3, 57-64.

Schlichting, H. (1979). Boundary layer theory, 7th edn. New York: McGraw-Hill.

Schofield, A. N. (1980). Cambridge geotechnical centrifuge operations. Géotechnique 30, No. 3, $227-268$.

Schofield, A. N. \& Wroth, C. P. (1968). Critical state soil mechanics, p. 52. New York: McGraw-Hill.

Taylor, D. W. (1948). Fundamentals of soil mechanics, p. 113. New York: Wiley.

Terzaghi, K. (1943). Theoretical soil mechanics, pp. 237-238. New York: Wiley.

Yih, C. S. (1977). Fluid mechanics, pp. 375-379. Ann Arbor: West River Press. 